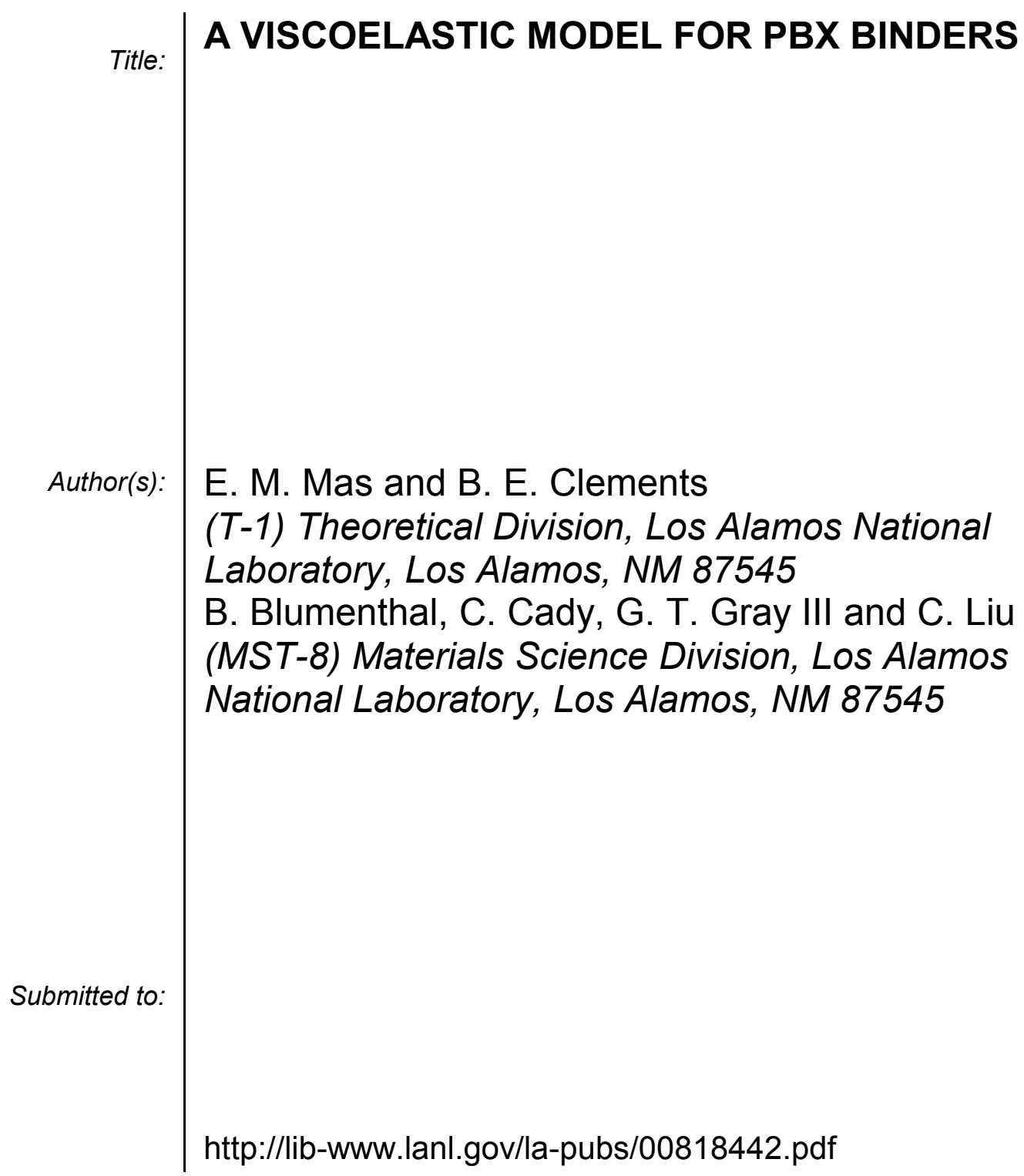

Los Alamos National Laboratory, an affirmative action/equal opportunity employer, is operated by the University of California for the U.S. Department of Energy under contract W-7405-ENG-36. By acceptance of this article, the publisher recognizes that the U.S. Government retains a nonexclusive, royaltyfree license to publish or reproduce the published form of this contribution, or to allow others to do so, for U.S. Government purposes. Los Alamos National Laboratory requests that the publisher identify this article as work performed under the auspices of the U.S. Department of Energy. Los Alamos National Laboratory strongly supports academic freedom and a researcher's right to publish; as an institution, however, the Laboratory does not endorse the viewpoint of a publication or guarantee its technical correctness. 


\title{
A VISCOELASTIC MODEL FOR PBX BINDERS
}

\author{
E. M. Mas and B. E. Clements \\ (T-1) Theoretical Division, Los Alamos National Laboratory, Los Alamos, NM 87545 \\ B. Blumenthal, C. Cady, G. T. Gray III and C. Liu \\ (MST-8) Materials Science Division, Los Alamos National Laboratory, Los Alamos, NM 87545
}

\begin{abstract}
Stress-strain measurements done at different rates and temperatures along with measurements of the rate- and temperature-dependent dynamic storage modulus have allowed us to construct a generalized Maxwell model for the linear viscoelastic response of plasticized estane. A theoretical analysis is presented to include effects of impurites.
\end{abstract}

\section{INTRODUCTION}

Complete knowledge of the thermo-mechanical behavior of the constituents of PBX-9501 is required for any micromechanics method to be a useful tool for modeling its behavior. The primary constituents of PBX 9501 are the explosive cyclotetramethylenetetranitramine (HMX) crystals and the inert plasticized estane binder matrix. Estane 5703 is a polyester polyurethane elastomer manufactured by the B.F. Goodrich Company with a density of 1.19 $\mathrm{gm} / \mathrm{cm}^{3}$. The polymeric binder shows dramatic sensitivity to changes in strain rates and temperatures. For example, a change in the temperature from $-50 \mathrm{C}$ to $50 \mathrm{C}$ will have an associated change in the shear modulus of five orders of magnitude. Obviously, a successful theory for PBX 9501 must account for this behavior. Because of recent experimental effort, much high-quality stress-strain data has become available for the plasticised binder. A primary goal was to use this data to formulate a generalized Maxwell model (GMM) thermo-mechanical constitutive law for the binder. While a GMM constitutive law has immediate applications for PBX 9501, our theoretical analysis used to obtain the constitutive law has interest to the general community involved with plastic bonded high explosives.
The aforementioned stress-strain data was measured by the LANL's Material Structure/Property Group (MST-8) and was obtained by several different experimental methods. An Intron 5567 testing machine was used for measuring uniaxial stressstrain data for rates in the range of $10^{-4} \mathrm{~s}^{-1}$ to $1 \mathrm{~s}^{-1} \mathrm{~A}$ hydraulic MTS 810 material testing machine was used for rates between $10^{-1} \mathrm{~s}^{-1}$ and $10 \mathrm{~s}^{-1}$, while Split-Hopkinson Pressure Bar (SHPB) was used for rates around $10^{3} \mathrm{~s}^{-1}$. Our approach is to use the torsion pendulum experiments of G. Flowers ${ }^{1}$ to construct the GMM. Upon determining the GMM we then use the Boltzman Superposition Principle (BSP) to calculate the stress-strain response predicted by our GMM for the binder. By comparing our stress-strain predictions with experiments involving Instron, MTS, and SHPB, which are uniaxial stress experiments, we can "fine tune" our GMM. Flowers' torsion pendulum data are functions of sinusoidal rather uniaxial loading strains. Consequently, a theoretical analysis is required to convert his data into a form useful for our micromechanics work.

\section{THEORETICAL ANALYSIS}

When the deformation is small, the theory of linear viscoelasticity assumes that the dynamic behavior of any viscoelastic material can be described in terms of 
various relaxation processes. These dynamic processes originate from the motions that occur in any complicated entangled molecular network comprising the viscoleastic material. Since the number of relaxation processes are typically huge, a continuous distribution of relaxation times is required to fully describe the dynamic behavior of the material. The corresponding transient shear moduli can be expressed in terms of this relaxation time distribution as:

$$
G(t)=G_{e}+\int_{-\infty}^{+\infty} H(\tau) e^{-t / \tau} d(\ln \tau)
$$

where $H(\tau) d(\ln \tau)$ represents the relaxation times in the range $\ln \tau$ to $\ln \tau+d(\ln \tau)$, and $G_{e}$, if different from zero, is called the equilibrium modulus.

The Generalized Maxwell Model of viscoelasticity replaces the spectrum of relaxation times with a discrete spectrum labeled by $\tau_{i}$

$$
H(\tau)=\sum_{i=1}^{n} G_{i} \tau_{i} \delta\left(\tau-\tau_{i}\right)
$$

where $G_{i}$ is the strength of the $\mathrm{i}^{\text {th }}$ mode. In this approximation, the shear modulus becomes:

$$
G(t)=G_{e}+\sum_{i=1}^{n} G_{i} e^{-t / \tau_{i}}
$$

Flowers has determined the storage and loss moduli, $G^{\prime}(\omega)$ and $G^{\prime \prime}(\omega)$, respectively, for the binder over a temperature range of $-150 \mathrm{C}$ to $75 \mathrm{C}$, and driving frequencies from 0.6 radians s$s^{-1}$ to 60 radians $\mathrm{s}^{-1}$. To extend the effective frequency range, Flowers invoked the well-known time-temperature superposition theory of Williams, Landel and Ferry ${ }^{2}$ (WLF), by introducing a time-temperature shift factor $a_{T}$. Figure 1 shows a numerical fit to Flowers' measurements of $\log G^{\prime}$ as a function of $\log \left(a_{T} \omega\right)$, at a reduced temperature $T_{0}$ of $19 \mathrm{C}$.

Flowers also provided data on the temperature dependence of his shift function. We we fit his shift function to a WLF equation:

$$
\log \left(a_{T}\right)=-6.5 \frac{\left(T-T_{0}\right)}{120+T-T_{0}} .
$$

The storage modulus $G^{\prime}(\omega)$ is related to the shear relaxation function by

$$
G^{\prime}(\omega)=\omega \int_{0}^{\infty} d t^{\prime} \sin \left(\omega t^{\prime}\right) G\left(t^{\prime}\right)
$$

Thus

$$
\begin{aligned}
G^{\prime}(\omega) & =G_{e}+\int_{-\infty}^{+\infty} H(\omega) d(\ln \tau) \frac{\omega^{2} \tau^{2}}{1+\omega^{2} \tau^{2}} \\
& =G_{e}+\sum_{i=1}^{n} G_{i} \frac{\omega^{2} \tau_{i}^{2}}{1+\omega^{2} \tau_{i}^{2}}
\end{aligned}
$$

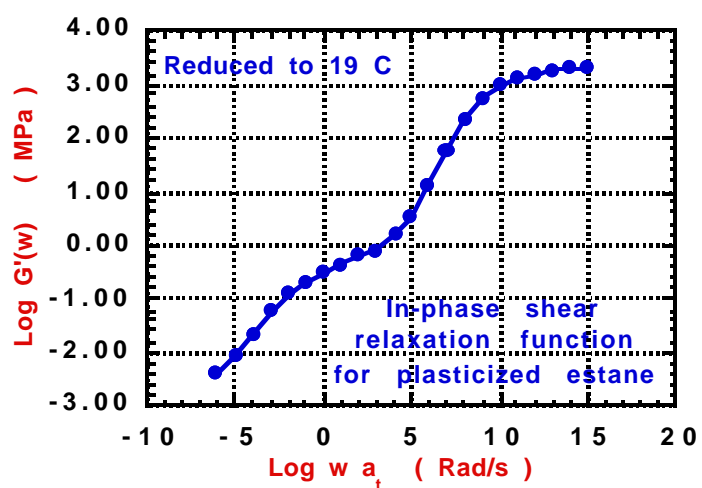

FIGURE 1. The storage moduli plotted against the driving frequency for the PBX 9501 binder.

From $G^{\prime}(\omega)$ we determine the $G_{i}$, for choice of relaxation times $\tau_{i}=1.5 a_{T} 10^{(7-i)}$ (in seconds). Since the coefficients in Eq. (6) are the same in those of Eq. (3) we simultaneously get the correct coefficients to use in our transient shear moduli.

\begin{tabular}{|l|l|l|l|}
\hline ELEMENT & LOGG $_{\mathrm{i}}$ & ELEMENT & LOG G \\
\hline 1 & -2.38 & 12 & 0.418 \\
\hline 2 & -2.13 & 13 & 1.11 \\
\hline 3 & -1.80 & 14 & 1.72 \\
\hline 4 & -1.42 & 15 & 2.35 \\
\hline 5 & -1.17 & 16 & 2.64 \\
\hline 6 & -1.05 & 17 & 2.66 \\
\hline 7 & -0.937 & 18 & 2.54 \\
\hline 8 & -0.847 & 19 & 2.40 \\
\hline 9 & -0.790 & 20 & 2.25 \\
\hline 10 & -0.654 & 21 & 2.07 \\
\hline 11 & -0.323 & 22 & 1.88 \\
\hline
\end{tabular}

TABLE 1. 22-element Maxwell model for plasticized estane. The units are MPa for $G_{i}$. 
We approximate the bulk modulus of the binder as the strain-rate independent value of $3.65 \mathrm{GPa}$. For the case of uniaxial stress, the relevant modulus is Young's, given by

$$
E(t)=\frac{9 K G(t)}{3 K+G(t)} \approx 3 G(t) .
$$

Having determined $G(t)$ from Eq. (3), we then determine the stress-strain behavior from

$$
\sigma(t)=\int_{0}^{t} E\left(t-t^{\prime}\right) \dot{\varepsilon}\left(t^{\prime}\right) d t^{\prime} .
$$

Our theoretical stress-strain curves fron Eq. (8) are compared to those measured at MST-8. A fine-tuned GMM was then constructed to improve the agreement (Table 1) although only small changes in the origianl GMM were required. The final stressstrain curves are compaired to experiment in Fig. 2.

\section{RESULTS}

Compressive stress-strain curves measured at MST-8, and those obtained by our theoretical analysis are shown in Fig. 2 for room temperature, low strain rates, and in Figs. 3, and 4 for higher strain rates and colder temperatures.

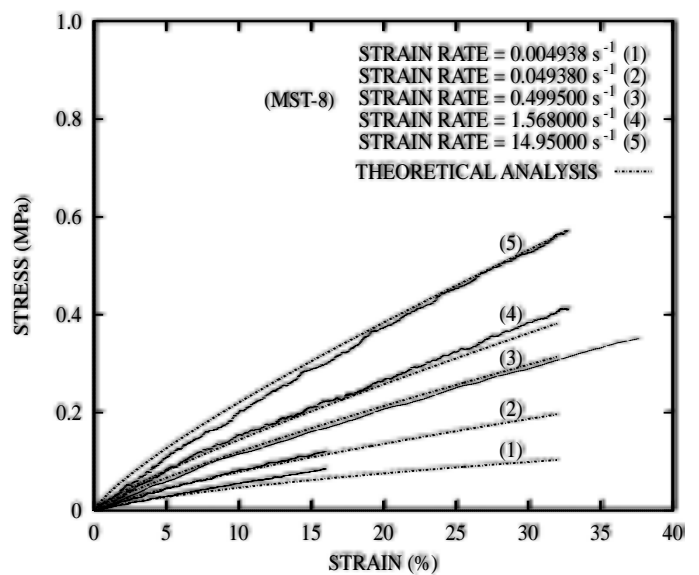

FIGURE 2. Room temperature, low strain-rate compression stress-strain curves for the plasticised binder. At each strain rate, two stress-strain curves are shown, one corresponding to direct stress-strain measurements taken at MST-8, and one corresponding to our theoretical analysis of Flowers' torsion pendulum measurements.

It is clear, with the exception of Fig. 4, that the agreement between our analysis and the MST- 8 data is very good. In these temperature and strain rate regimes our derived constitutive law using the GMM, plus the temperature dependence from the WLF equation (Eq. (4)) provide a good description of the thermo-mechanical behavior of the binder.
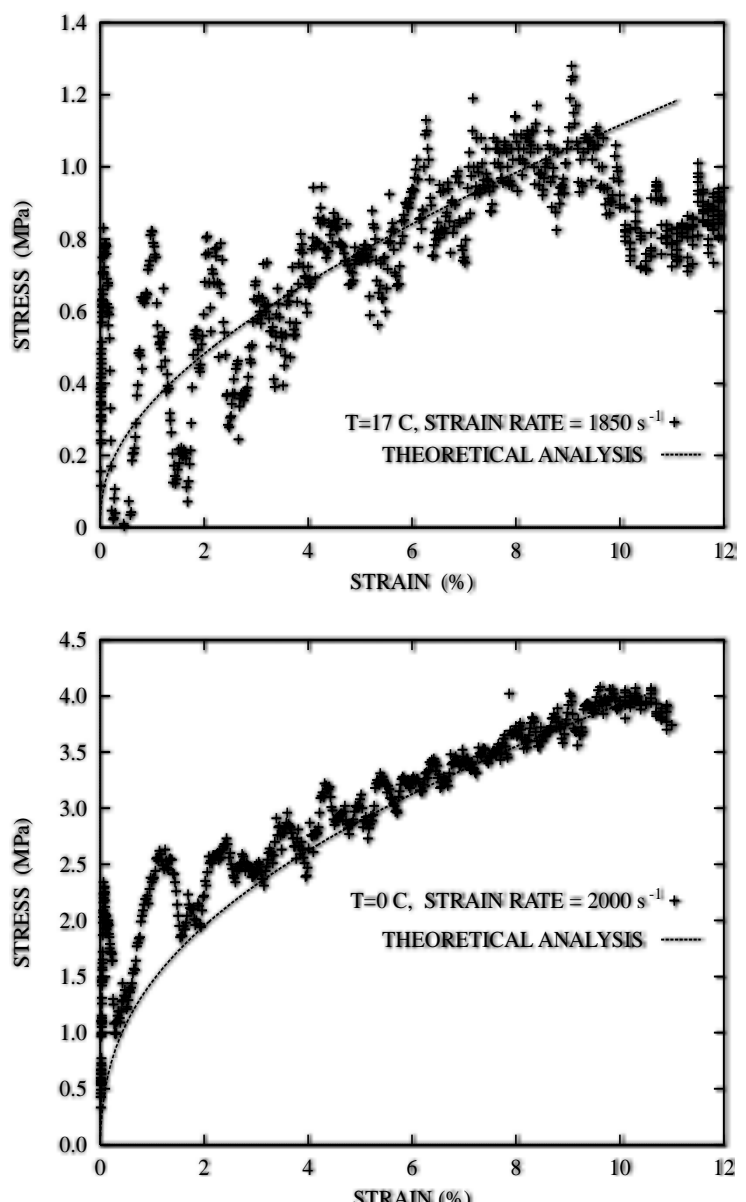

FIGURE 3. TOP: Split Hopkinson Pressure Bar stress-strain data taken at $17 \mathrm{C}$ and a strain rate of $1850 \mathrm{~s}^{-1}$. The dashed curve is our corresponding theoretical analysis of Flowers' torsion pendulum measurements. BOTTOM: Split Hopkinson Pressure Bar stress-strain data taken at $0 \mathrm{C}$ and a strain rate of $2000 \mathrm{~s}^{-1}$. The dashed curve is our corresponding theoretical analysis of Flowers' torsion pendulum measurements.

At low temperatures and high strain rates, however, the assumptions inherent in the construction of the binder constitutive law begin to fail, and the analysis yields strong discrepancies with the uniaxial SHPB data (Fig. 4). The likely cause of this discrepancy is that, at very low temperatures and high strain rates, the glass-like behavior of the binder has associated yielding which begins to 
dominate the stress-strain response, thus invalidating the assumptions of linear viscoelasticity.

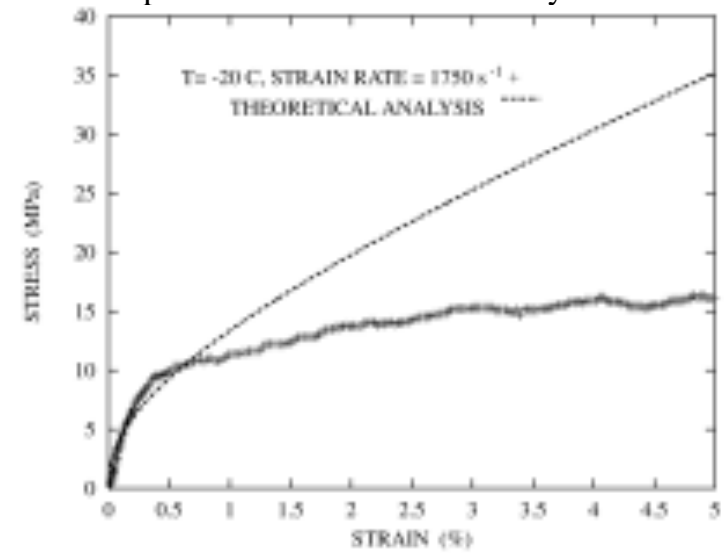

FIGURE 4. Split Hopkinson Pressure Bar stress-strain data taken at $-20 \mathrm{C}$ and a strain rate of $1750 \mathrm{~s}^{-1}$. The dashed curve is our corresponding theoretical analysis of Flower's torsion pendulum measurements. The plateau region in the Split Hopkinson Pressure Bar measurements indicate that the binder is in a regime where linear viscoelasticity begins to fail.

We have extended GMM for the binder to include elastic impurites. The entire composite will respond viscoelastically, obtaining its strain rate dependence from the matrix. As with the plasticized estane, we expect that the BSP will provide an adequate description for the time dependent stress field, $\sigma_{i j}(t)$, for the composite,

$$
\sigma_{i j}(t)=\int_{0}^{t} d t^{\prime} L_{i j k l}^{C}\left(t-t^{\prime}\right) \dot{\varepsilon}_{k l}\left(t^{\prime}\right)
$$

where $L_{i j k l}^{C}(t)$ is the stress relaxation function for the composite and $\dot{\varepsilon}(t)$ is the strain rate tensor. $L_{i j k l}^{C}(t)$ can be expressed as

$$
\begin{aligned}
L_{i j k l}^{C}(t)= & {\left[K^{C}(t)-\frac{2}{3} G^{C}(t)\right] \delta_{i j} \delta_{k l} } \\
& +G^{C}(t)\left[\delta_{i k} \delta_{j l}+\delta_{i l} \delta_{j k}\right],
\end{aligned}
$$

where $K^{C}(t)$ and $G^{C}(t)$ are the composite bulk and shear relaxation functions, respectively. Our derivation of these two functions begins with the theory of Weng and coworkers ${ }^{3}$. These authors generalized Eshelby-Mori-Tanaka theory to include linear viscoelastic materials.

After a rather length analysis we are able to express the composite moduli in a modified GMM.
The coefficients are now functions of the bulk moduli and concentrations of the binder and the impurites, $K^{m}, \mathrm{c}_{\mathrm{m}}$ and $K^{f}, \mathrm{c}_{\mathrm{f}}$ respectively. Note, that unlike the pure binder, the composite now has a time-dependent bulk relaxation function. The result of our analysis gives:

$$
\begin{aligned}
& K^{C}(t)=K_{0}^{C}+\sum_{i=1}^{M} K_{i}^{C} e^{-t / \tau_{i}} \\
& G^{C}(t)=G_{0}^{C}+\sum_{i=1}^{M} G_{i}^{C} e^{-t / \tau_{i}},
\end{aligned}
$$

where the coefficients, $K_{i}^{C}$ and $G_{i}^{C}$, are given by

$$
\begin{aligned}
& K_{i}^{C}= \begin{cases}K^{f} K^{m}\left(c_{f} K^{m}+c_{m} K^{f}\right)^{-1} & i=0 \\
\frac{4}{3} c_{f} c_{m}\left(K^{f}-K^{m}\right)^{2} * & i \neq 0 \\
\left(c_{f} K^{m}+c_{m} K^{f}\right)^{-2} G_{i}^{m}\end{cases} \\
& G_{i}^{C}=\left\{\begin{array}{cc}
0 & i=0 \\
\left(1+\frac{5 c_{f}}{2 c_{m}}\right) G_{i}^{m} & i \neq 0
\end{array}\right.
\end{aligned}
$$

where $G_{i}^{m}$ are the coefficients of the GMM for the unfilled binder provided in Table 1. This analysis has be extended to the general case of ellipsoidal shaped impurities.

\section{ACKNOWLEDGMENTS}

The authors gratefully acknowledge financial support for this work from the Joint DoD/DOE Munitions Technology Development. Research is supported by the USDOE under contract W-7405ENG-36.

\section{REFERENCES}

1) Flowers, G. L., Characterization of binders used in high explosives and the effect of binder strength on impact sensitivity of explosives. Report MHSMP-854, Pantex, August 1985

2) Ferry, J. D., Viscoelastic Properties of Polymers (2nd Ed., Wiley, New York, 1970).

3). Li, J., and Weng, G. J., Mech. Mater. 22 179-188 (1995); Wang, Y.M., and Weng, G. J., J. Appl. Mech., 59, 510-518 (1992). 